\title{
Analyses écologique et structurale de la forêt communautaire de Kaodji au Bénin
}

\author{
Julien DJEGO ${ }^{2^{*}}$, Moussa GIBIGAYE ${ }^{1}$, Brice TENTE $^{1}$ et Brice SINSIN $^{2}$ \\ ${ }^{1}$ Département de Géographie et Aménagement du Territoire/FLASH/UAC, Bénin. \\ ${ }^{2}$ Laboratoire d'Ecologie Appliquée/FSA/UAC. 01 BP : 526 Cotonou, Bénin. \\ *Auteur correspondant, E-mail : gdjego@yahoo.fr
}

\section{RESUME}

La forêt communautaire de Kaodji au Bénin regorge d'importantes ressources biologiques, qui ne sont pas encore toutes bien connues. Cette étude examine les caractéristiques écologique et structurale de cette forêt. A cet effet, la diversité floristique a été estimée au moyen de 48 relevés floristiques effectués selon la méthode sigmatiste de Braun-Blanquet (1932) dans différentes strates forestières et d'indices de diversité. Des mesures dendrométriques, de distances et de pentes ont été effectuées en vue de déterminer le profil structural lié à chaque type de phytocénoses et celui topographique de la forêt de Kaodji. Cette forêt se remarque notamment par deux strates nettement indépendantes (indice de Jaccard <0,45). Il s'agit de : (i) la savane arbustive ayant pour espèces dominantes, Combretum collinum et Daniella oliveri; (ii) et la forêt claire marquée par Isoberlinia doka et Combretum collinum. Leurs richesses floristiques moyennes sont respectivement de 36 et de 49. Les indices de Shannon et l'équitabilité de Piélou montrent des valeurs relativement faibles (2,33 bits et 0,45 en savane et 2,69 bits et 0,48 en forêt claire). La répartition par classe de diamètre des arbres dans ces deux milieux présente l'allure d'un « $\mathbf{J}$ » renversé et s'ajuste au mieux à une fonction logarithmique. Sur le plan topographique, cette forêt présente un relief vallonné d'allure pittoresque pouvant constituer un site touristique en cas de conservation et d'aménagement.

(C) 2012 International Formulae Group. All rights reserved.

Mots clés : Forêt communautaire, topographie, phytodiversité, Kaodji, Bénin.

\section{INTRODUCTION}

La diversité biologique au Bénin est le fait d'évènements paléo-climatiques majeurs ayant conduit au fait établi de Dahomey Gap, une interruption de la ceinture forestière allant du bassin congolais au bassin du Niger plus généralement connu sous le nom de bloc guinéen. Dans ce couloir sec dahoméen, subsistent encore quelques îlots de forêts telles que la forêt dense semi-décidue de la Lama (Paradis et Houngnon, 1977 ; Djègo et
Oumorou, 2009) et la forêt claire de Kaodji. Ces écosystèmes du Bénin renferment une diversité faunique et floristique non négligeable allant des invertébrés aux mammifères y compris les oiseaux, reptiles et poissons, et bien d'autres espèces (Sinsin et al., 2004). L'inventaire des connaissances sur la diversité biologique a bien prouvé le potentiel biologique du Bénin. Les études d'expertise sur le statut des espèces de faune mammalienne menacées en 2002 , et bien 
d'autres études scientifiques sur les menaces pesant sur la diversité biologique (Sinsin et al., 2010) ont bien démontré les risques de disparition que court une bonne partie de cette diversité biologique aux plans local et national. Et pour cause, les populations vivent en majorité de ces ressources naturelles pour de multiples raisons (usages alimentaire, commercial, socioculturel, industriel, etc.). On y enregistre une exploitation non contrôlée de certaines espèces de faune et de végétation. Nombreuses d'entre elles sont devenues vulnérables, rares, menacées d'extinction ou ont disparu de certains écosystèmes. Leur habitat (formation végétale) s'amenuise du fait de la volonté obstinée des paysans d'étendre démesurément les superficies cultivées pour satisfaire non seulement les besoins alimentaires, mais également pour se procurer des ressources financières immédiates (cultures de rentes). Ceci étant, le taux de déboisement en Afrique tropicale et particulièrement au Bénin reste encore élevé malgré la diminution du taux de perte nette de forêts, passant de 4 millions d'ha/an pendant la période de 1990 à 2000, à 3,5 millions d'ha/an entre 2000 et 2010 (FAO, 2011).

Des approches globales de gestion forestière, comme la gestion des écosystèmes et l'aménagement des sites, sont de plus en plus acceptées et mises en pratique. Ces approches reconnaissent le dynamisme des systèmes écologiques et sociaux, la nécessité d'une gestion adaptative et l'importance des prises de décisions collectives. Les stratégies intégrées de conservation des forêts dans lesquelles la sauvegarde des ressources forestières en général et de la biodiversité en particulier, nécessitent en premier lieu un bon état des lieux. Au moment où la préservation de l'environnement et la sauvegarde de la diversité biologique sont prônées de par le monde, il serait alors impérieux de prendre des mesures visant à ralentir le phénomène de dégradation de la biodiversité, et donc à favoriser leur gestion durable. Cette gestion nécessite d'abord, une bonne connaissance de la composition floristique, de la structure et de la dynamique de régénération (Sokpon, 1995) des écosystèmes menacés. Dans cette optique, cette étude s'intéresse à la forêt communautaire de Kaodji afin de contribuer à l'amélioration de sa connaissance pour une utilisation rationnelle et durable des ressources qu'elle regorge.

L'objectif général est d'évaluer les potentiels floristique et ligneux de ces forêts en vue d'une meilleure prise de décision. De façon spécifique, il s'agira de déterminer la diversité floristique des ces formations végétales; d'analyser leurs caractéristiques écologiques et dendrométriques et enfin de déterminer le profil topographique de la zone agro-écologique de Kaodji.

\section{MATERIEL ET METHODES Milieu d'étude}

Le milieu d'étude est situé au centre du Bénin. Il est constitué par la forêt communautaire de Kaodji encore appelée massif forestier de Zou-Zounkan. Il s'étend sur une superficie totale de 36119 hectares et se situe entre les communes de Zangnanado à l'Est (Banamè); de Covè au Sud (Naogon) ; de Dassa-Zoumè au Nord (Paouignan) et la rivière Zou à l'Ouest. La forêt est comprise entre $7^{\circ}$ et $7^{\circ} 30$ ' latitude nord et $2^{\circ} 15^{\prime}$, et $2 \circ 30$ ' longitude Est. Le climat de ce milieu est de type intermédiaire entre le climat subéquatorial maritime et le climat soudano guinéen. Il est caractérisé par quatre saisons et un régime pluviométrique de type bimodal. Les sols sont de types ferrugineux et ferralitiques (ORSTOM, 1968). On y note la présence de cuirasse latéritique. La végétation est composée de savane et de forêt claire. Cette forêt abrite le site écologique du trou ou la source aux phacochères Hanto-dogban. L'aménagement de ce massif se fait actuellement par le Projet Bois de Feu Phase II (PBFII) avec la participation très active des populations riveraines. Le massif est subdivisé en neuf (9) unités d'aménagements qui 
associent 17 villages riverains. Les groupes socioéconomiques majoritaires sont les Mahi, Fon et Holli.

\section{Matériel}

Le matériel de cette étude est constitué par la forêt communautaire de Kaodji.

\section{Méthodes de Collecte de données Données floristiques}

La diversité spécifique rend compte de la richesse et de la distribution d'abondance spécifique des phytocénoces (Danais, 1982). Elle est définie par la richesse spécifique et l'indice de diversité de Shannon (Chaneton et al., 1997).

Pour estimer la diversité biologique des formations végétales étudiées, 48 relevés phytosociologiques ont été effectués selon la méthode sigmatiste de Braun-Blanquet (1932). Toutes les espèces végétales présentes ont été recensées et affectées chacune d'un coefficient d'abondance-dominance suivant l'échelle ci-dessous axée sur le recouvrement moyen (RM) proposée par Braun-Blanquet (1932) :

$>$ 5: espèces couvrant 75 à $100 \%$ de la surface du relevé soit un RM de $87,5 \%$;

$>$ 4: espèces couvrant 50 à $75 \%$ de la surface du relevé soit un RM de $62,5 \%$;

$>$ 3: espèces couvrant 25 à $50 \%$ de la surface du relevé soit un RM de 37,5\% ;

$>$ 2: espèces couvrant 5 à $25 \%$ de la surface du relevé soit un RM de $15 \%$;

$>$ 1: espèces couvrant 1 à $5 \%$ de la surface du relevé soit un RM de $3 \%$ et;

$>$ +: espèces couvrant moins de $1 \%$ de la surface du relevé soit un RM de $0,5 \%$.

Au total, 48 relevés ont été effectués dans chaque formation végétale et la superficie des placeaux est de $900 \mathrm{~m}^{2}$.

\section{Données dendrométriques}

Le diamètre de tous les ligneux ayant un $\mathrm{dbh}_{1,30} \geq 10 \mathrm{~cm}$, a été mesuré dans les placeaux installés en forêt et en plantation dans la forêt classée de la Lama, tout comme en forêt claire à Kaodji. En savane par contre, compte tenu de la faible densité des arbres, seuls ceux ayant un $\mathrm{dbh}_{1,30} \geq 5 \mathrm{~cm}$ ont été pris en compte.

\section{Détermination de profil topographique}

Un transect a été réalisé sur une distance de $1000 \mathrm{~m}$ linéaire suivant l'azimut $60^{\circ}$. De part et d'autre sur une bande de $5 \mathrm{~m}$ de la ligne de marche, un inventaire des ligneux a été fait. Tous les individus ayant un diamètre à hauteur d'homme (DBH) supérieur ou égale à $10 \mathrm{~cm}$ ont été relevés. Des mesures de distances et de pentes ont été faites et ont permis de réaliser la coupe écofloristique de la forêt de Kaodji.

\section{Analyse des données \\ Diversité floristique}

La diversité spécifique des différentes formations végétales est évaluée à l'aide des richesses spécifiques observées et estimées. De même, plusieurs indices ont été calculés : les indices de diversité de Shannon $(\mathrm{H})$, de Simpson (1/D) et de Berger-Parker (1/d); l'équitabilité de Piélou (E) et la richesse spécifique effective $(\mathrm{N})$.

Le nombre probable d'espèces présentes dans chaque formation végétale a été calculé par la méthode de Jack-Knife. Le nombre d'espèces estimé $(\hat{\mathrm{S}})$ est :

$$
\hat{\mathrm{S}}=s+\frac{(n-1)}{n} K ; \mathrm{s}=\text { nombre }
$$

d'espèces observées dans les placeaux; $n=6$ (nombre de placeaux); $\mathrm{K}=$ nombre d'espèces uniques.

L'intervalle de confiance de $\hat{\mathrm{S}}$ est calculé par la formule :

$$
\mathrm{IC}=\hat{\mathrm{S}} \pm t_{0,025}(5) \sqrt{\operatorname{Var}(\mathrm{S})}
$$

La variance de $\hat{\mathrm{S}}[\operatorname{Var}(\hat{\mathrm{S}})]$ est donnée par :

$\operatorname{Var}(\hat{\mathrm{S}})=\frac{(n-1)}{n}\left[\sum_{i=1}^{j}\left(j^{2}{ }_{i} f_{j}\right)-\frac{K^{2}}{n}\right] ;$

j désigne les classes de placeaux selon leur effectif en espèces uniques ; $f_{j}$ est le nombre de placeaux ayant $\mathrm{j}$ espèces uniques. 
L'indice de diversité de Shannon noté $\mathrm{H}$ et exprimé en bits est calculé par la formule :

$$
\mathbf{H}=-\sum p i . \log _{2} p i \quad \text { avec }
$$

$p i=\frac{n i}{\sum n i} ; \mathrm{Pi}=$ fréquence relative des

individus de l'espèce $\mathrm{i}$; ni = recouvrement moyen des individus de l'espèce $\mathrm{i} ; \mathrm{n}=$ recouvrement total des individus de la formation végétale. Il permet d'évaluer la diversité des espèces végétales. La diversité est faible lorsque $\mathrm{H}<3$ bits, moyenne si $\mathrm{H}$ est compris entre 3 et 4 bits, puis élevée quand $\mathrm{H}$ $\geq 4$ bits. Le milieu est peu diversifié quand $\mathrm{H}$ est faible et relativement diversifié en espèces quand $\mathrm{H}$ est fort.

L'équitabilité de Pielou (E) correspond au rapport entre la diversité obtenue et la diversité maximale. Il exprime la régularité ou l'équitable répartition des individus au sein des espèces. Il est donné par la formule :

$E=\frac{H}{\log _{2} S} ; \quad \mathrm{S}$ désigne la richesse spécifique.

L'équitabilité est faible lorsque $\mathrm{Eq}<$ 0,6 ; moyenne quand $\mathrm{Eq}$ est compris entre 0,6 et 0,8 et élevée si $\mathrm{Eq} \geq 0,8$. Si E est faible, on conclut que peu d'espèces concentrent la majorité des individus du milieu. Lorsque $\mathrm{E}$ est élevé, on déduit que le milieu n'est pas spécialisé et donc les individus sont bien répartis au sein des espèces.

$\mathrm{Si} \mathrm{H}$ et $\mathrm{E}$ sont faibles, alors le milieu est homogène et spécialisé. Si H et E sont élevés, alors le milieu est isotrope.

La richesse spécifique effective $(\mathrm{N})$ indique le nombre d'espèces responsables de la diversité observée. Elle est donnée par la formule:

$$
N=2^{H} ; 2 \text { désigne la base du }
$$
logarithme utilisé pour calculer l'indice de diversité de Shannon $\mathrm{H}$.

L'indice de dominance de Simpson (D) rend compte du biais induis par l'abondance de certaines espèces. Il est calculé par la relation:

$$
D=\sum_{i=1}^{S} P_{i}^{2} ; \quad \mathrm{Pi}=\text { fréquence }
$$

relative des individus de l'espèce i. Son inverse 1/D exprime la diversité et varie de 1 à la diversité spécifique $S$.

L'indice de dominance de BergerParker (d), est calculé par:

$$
d=\frac{N_{\max }}{N} ; \mathrm{N}_{\max }=\text { recouvrement }
$$

de l'espèce la plus abondante; $\mathrm{N}=$ recouvrement total de toutes les espèces. Son inverse (1/d) désigne l'indice de diversité selon Berger-Paker.

Pour comprendre les relations entre les différentes formations végétales, l'indice de similitude de Jaccard $\left(\mathrm{I}_{\mathrm{J}}\right)$ est calculé par la formule :

$$
I_{J}=100 \times \frac{c}{a+b-c} \text {; où a et b }
$$

désignent respectivement le nombre d'espèces des milieux $\mathrm{A}$ et $\mathrm{B}$ et $\mathrm{c}$ le nombre d'espèces communes aux deux milieux. Si $\mathrm{I}_{\mathrm{J}}>50 \%$, les milieux sont similaires et si $\mathrm{I}_{\mathrm{J}}<50 \%$, il y a dissimilitude entre les milieux. En pratique quand $\mathrm{I}_{\mathrm{J}}>45 \%$, on admet qu'il y a similitude entre les milieux concernés.

\section{Caractéristiques structurales}

La surface terrière de la formation végétale est la somme des surfaces terrières de tous les arbres qui la constituent. Elle est obtenue par la formule :

$$
\mathbf{G}=\Sigma \pi \mathbf{D}^{2} / \mathbf{4} ; \text { avec } \mathrm{G}=\text { surface }
$$
terrière exprimée en $\mathrm{m}^{2} /$ ha et $\mathrm{D}=$ diamètre à hauteur de poitrine d'homme des arbres.

La densité des arbres d'une formation végétale $(\mathrm{N})$ est le nombre de tiges par hectare de cette formation végétale. Elle est calculée pour les arbres et arbustes à $\mathrm{dbh} \geq 10 \mathrm{~cm}$.

La représentation par classe de diamètre des arbres a été réalisée pour élucider la structure diamétrique en plantation et en forêt naturelle. 


\section{RESULTATS}

\section{Diversité floristique des formations végétales}

$\mathrm{Au}$ total, 83 espèces réparties en 52 familles ont été recensées. La richesse spécifique (RS) varie d'une formation végétale à une autre (Tableau 1) et est relativement plus faible en savane (36 espèces) qu'en forêts claires (49 espèces).

Les valeurs des indices de diversité sont plus faibles en savane qu'en forêt claire. Les valeurs de l'indice de Shannon et de l'équitabilité de Piélou permettent de conclure que les savanes et forêt claire représentent un milieu homogène et spécialisé. L'effet de dominance observée dans la forêt claire est exercé par 7 espèces dont deux recouvrent plus de $77,5 \%$ de la végétation. Il s'agit de Isoberlinia doka $(62,5 \%)$ et Combretum collinum (15\%). En savane, 6 espèces dominent, notamment Combretum collinum $(50,17 \%)$ et Daniella oliveri $(25 \%)$. Les valeurs de l'indice de Berger-Parker montrent que le recouvrement relatif de l'espèce la plus dominante (Combretum collinum) est plus élevé en savane qu'en forêt claire.

\section{Comparaison entre les différents écosystèmes}

L'analyse des indices de similarité de Jaccard entre la forêt claire et la savane arbustive indique une valeur inférieure à $45 \%$ $(25 \%)$; ce qui traduit une indépendance totale entre ces deux formations.

\section{Caractéristiques dendrométriques des formations végétales}

La surface terrière des ligneux matures ainsi que leur nombre à hectare sont résumés dans le Tableau 4 ci-dessous.

De l'analyse de ce tableau, il ressort que la surface terrière des savanes est très faible $\left(4,3 \mathrm{~m}^{2} / \mathrm{ha}\right)$. Cette valeur traduit réellement le faible potentiel en ligneux des milieux savanicoles.
Ces milieux regorgent un pourcentage important d'arbres de faible diamètre (compris entre 5 et $10 \mathrm{~cm}$ ). Au niveau des forêts claires, la valeur de la surface terrière de $11,11 \mathrm{~m}^{2} /$ ha est élevée par rapport à celle observée en savane. En ce qui concerne les densités d'arbres, elles sont plus élevées en savane qu'en forêt claire. Les Figures 1 et 2 cidessous traduisent la répartition par classe de diamètre des arbres dans les savanes et forêts claires. Elles présentent l'allure d'un « J » renversé et s'ajustent au mieux à une fonction logarithmique d'équations respectives $\mathrm{y}=$ $33,13 \ln (X)+56,254$ avec $\mathrm{R}^{2}=89,76 \%$ et $\mathrm{y}$ $=-17,61 \ln (x)+38.00$ avec $R^{2}=95,4 \%$. Cette allure est caractéristique des formations naturelles.

\section{Profil topographique de la zone agro- écologique de Kaodji}

Le transect réalisé du sommet au bas de pente sur le site de Kaodji nous a permis de tracer le profil topographique ci-dessous (Figure 3). Ce tracé montre un profil topographique associé aux différentes formations végétales.

Sur le plan topographique, le profil fait ressortir un type de relief représentant un plateau. Ce plateau est entaillé par deux vallées dont la plus importante est représentée par un cours d'eau. Ces deux vallées sont reliées par un interfluve. Le relief est dominé par deux formations végétales réparties sur des facettes topographiques précises: le sommet du plateau et le versant sont dominés par une formation mixte (herbacée-ligneux) mais à dominance d'herbacées. Le bas de versant et l'interfluve sont en majorité occupés par des ligneux. Le paysage observé s'explique par les activités anthropiques qui influent sur le sommet du plateau. Au niveau du sommet, s'exercent les activités agricoles qui participent à la dégradation du couvert végétal. Ainsi, la savane arbustive fait place à la savane herbeuse. Ce qui explique 
l'abondance des herbacées au niveau du sommet. Sous l'effet de l'érosion, les éléments nutritifs sont transportés du sommet vers le bas de versant où ils s'accumulent. A ce niveau, le sol est riche, ce qui favorise le développement des espèces ligneuses. De plus, le bas de versant est proche d'un cours d'eau où l'humidité permanente favorise le développement des espèces ligneuses (forêt galerie).

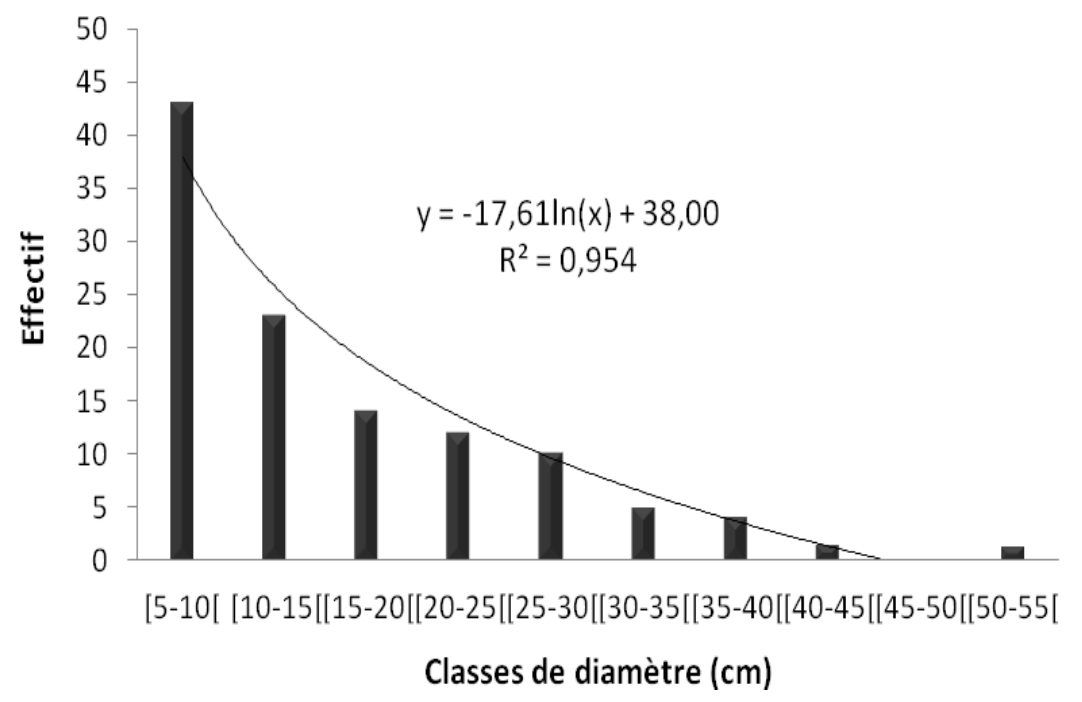

Figure 1: Répartition par classes de diamètre des arbres de savane.

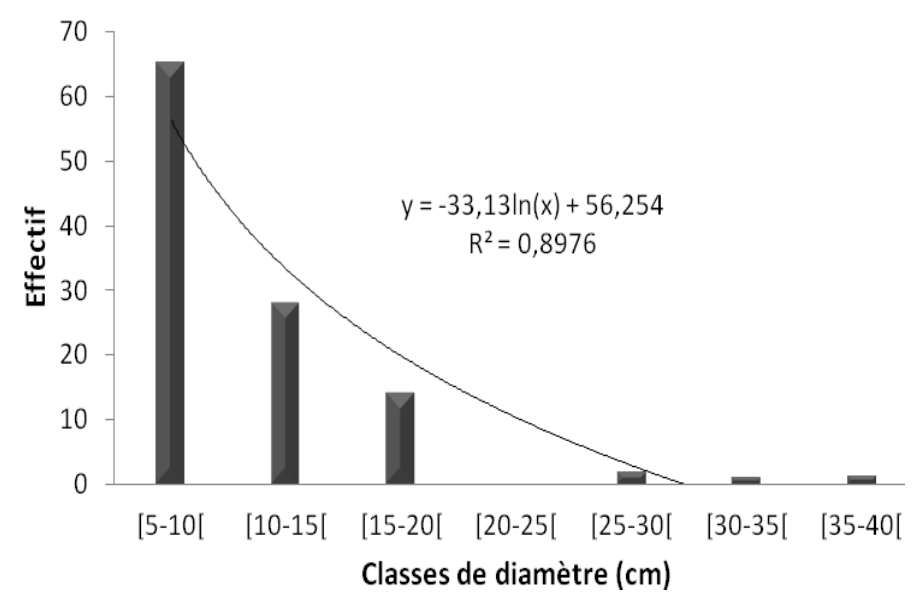

Figure 2: Répartition par classes de diamètre des arbres de forêt claire. 


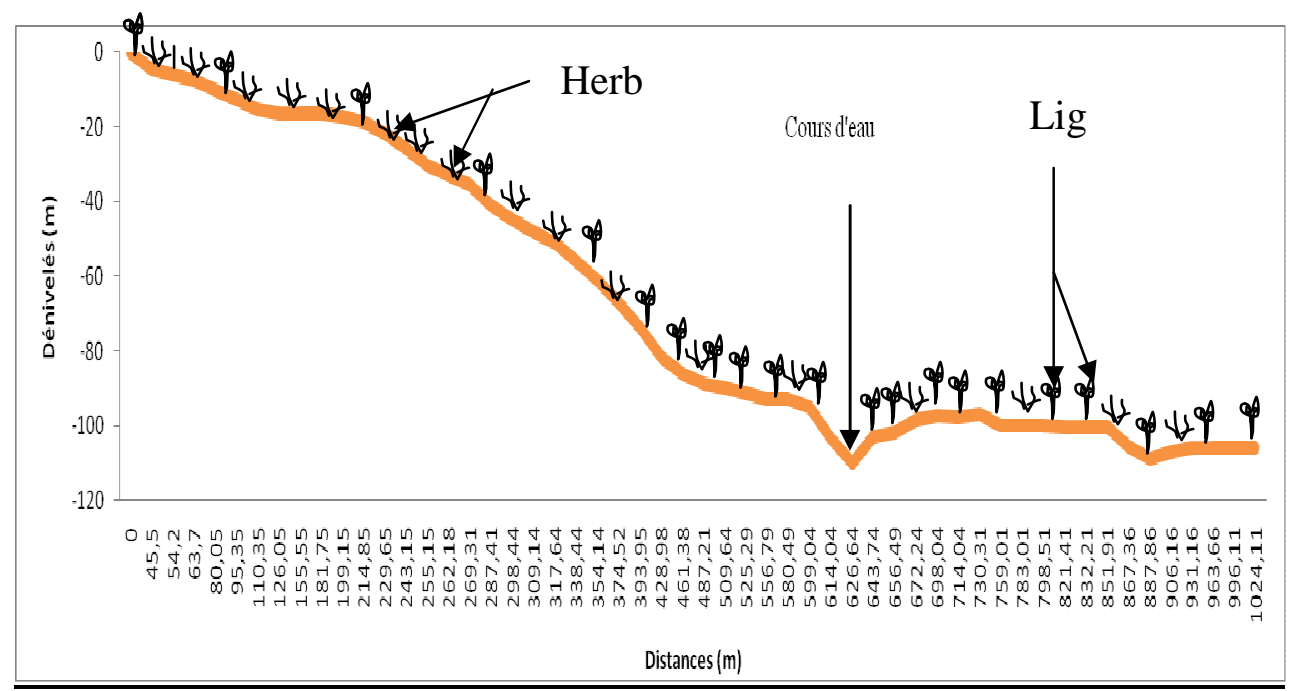

Figure 3: Profil topographique du site de Kaodji.

Tableau 1: Diversité floristique des formations végétales.

\begin{tabular}{ccc}
\hline Formations végétales & RS & RS estimée \\
\hline Savane & 36 & $52 \pm 10$ \\
Forêt claire & 49 & $68 \pm 12$ \\
Total & 238 & - \\
\hline
\end{tabular}

Tableau 2: Indices de diversité des formations végétales.

\begin{tabular}{ccccc}
\hline $\begin{array}{c}\text { Formations } \\
\text { végétales }\end{array}$ & $\begin{array}{c}\text { Shannon }(\mathbf{H}, \\
\text { bits) }\end{array}$ & Piélou (E) & Simpson (1/D) & $\begin{array}{c}\text { Berger- } \\
\text { Parker (1/d) }\end{array}$ \\
\hline Savane & 2,33 & 0,45 & 3,38 & 2,14 \\
Forêt claire & 2,69 & 0,48 & 2,68 & 1,66 \\
\hline
\end{tabular}

Tableau 3 : Densité des arbres et surfaces terrières des formations végétales.

\begin{tabular}{|c|c|c|c|c|}
\hline $\begin{array}{l}\text { Formations } \\
\text { végétales }\end{array}$ & $\begin{array}{l}\text { Densité } \\
\text { (tiges/ha) }\end{array}$ & des & arbres & Surface terrière $\left(\mathrm{m}^{2} / \mathrm{ha}\right)$ \\
\hline Savane & & 403 & & 4,3 \\
\hline Forêt claire & & 418 & & 11,11 \\
\hline
\end{tabular}




\section{DISCUSSION}

La physionomie et la structuration des formations végétales résultent d'une confluence de facteurs environnementaux (sol, climat, topographie, etc.) qui déterminent leur répartition dans les zones écologiques du monde. C'est ainsi que, pour des auteurs comme Lacoste et Roux (1972), Djègo (2006), Djègo et Oumorou (2009), les groupements végétaux sont l'expression de la synthèse des facteurs écologiques du milieu. La savane arbustive et la forêt claire observées à Kaodji en constituent une preuve. Situées dans la même zone biogéographique, ces formations présentent une certaine indépendance du point de vue structure et composition floristique. Cette diversité de milieux, résultant de l'interaction de facteurs environnementaux assure donc la répartition des phytocénoses. Selon Akpo et al. (1999), arbre et topographie s'associent pour créer une hétérogénéité de plus en plus grande du milieu qui gère la répartition de la végétation herbacée sous et hors couvert des arbres. Alors, la variation observée dans la composition et la richesse floristiques des savanes arbustives et forêts claires de Kaodji concorde avec ces résultats. Les valeurs de l'indice de Shannon $(\mathrm{H})$ et le coefficient d'équitabilité de Pielou sont utilisées pour mieux apprécier la diversité spécifique du sous-bois. L'examen de ces valeurs obtenues en savane et forêt claire de Kaodji, comparées aux valeurs obtenues en forêts denses semidécidues (Djègo, 2009) fait apparaitre que la végétation des forêts denses possède la diversité $\mathrm{H}$ et l'équitabilité (EQ) les plus fortes par rapport à celles des formations ouvertes. Il y a donc une plus grande diversité en milieu forestier dense. Selon Akpo et al. (1999), une plus grande diversité implique une plus grande égalité des contributions individuelles. Inversement, une diversité plus faible signifie une faible régularité de la répartition des individus.

La plus forte valeur de la surface terrière observée en forêt claire par rapport à celle obtenue en savane s'explique par un développement plus ou moins meilleur des arbres à cause des conditions écologiques de ce milieu. En effet, la zone de forêt claire est plus humide que la savane, ce qui représente un facteur essentiellement important pour le bon développement des arbres. La forte densité des arbres en savane s'explique par l'ouverture de la strate arbustive des savanes qu'en forêts claires. Cette ouverture favorise le développement d'autres ligneux par le biais de la pénétration des rayons solaires au sol, ce qui n'est pas le cas en forêt claire où il existe une concurrence en lumière des espèces de l'étage dominant. Le relief vallonné que présente la forêt de Kaodji est source de divers biotopes à l'origine d'une diversité biologique insoupçonnée. Le trou aux phacochères retrouvé sur le site en est une illustration. La mise en ouvre d'un plan d'aménagement adéquat de cette forêt permettrait de mettre en valeur ses potentialités biologiques, écologiques et touristiques. Tel est le cas de la forêt à Drimys brasiliensis au Brésil (Mariot et al., 2007). L'implication des populations riveraines à une telle perspective permettrait une gestion durable des ressources biologiques du milieu.

\section{Conclusion}

Les caractéristiques écologiques, structurales et topographiques que présente la forêt communautaire de Kaodji sont très édifiantes et méritent une attention particulière de la part des populations riveraines et des autorités en charge de la protection de la nature à divers niveaux. Au moment où la préservation de l'environnement et la sauvegarde de la diversité biologique sont prônées de par le monde, il serait impérieux de prendre des mesures visant à ralentir le phénomène de dégradation de la biodiversité, et donc à favoriser sa gestion durable. Le relief vallonné que présente le paysage de cette forêt communautaire et le site écologique du trou ou la source aux phacochères, sont des gîtes potentiellement riches en biodiversité mais aussi des sources exploitables en matière d'écotourisme et de 
pédagogie (laboratoire servant de lieu d'application et d'étude de cas aux étudiants).

\section{REFERENCES}

Akpo LE, Grouzis M, Bada F, Pontanier CF. 1999. Effet du couvert ligneux sur la structure de la végétation herbacée de jachères soudaniennes. Note originale. Sécheresse, 10(4): 253-61.

Braun-Blanquet J. 1932. Plant Sociology. Study of Plant Communities. Mc Cray Hill: New York, USA; London, UK; 439p.

Channeton E, Enrique J, Omacini M, Leon RJC. 1997. Plant diversity in relation to grazing, topography and scale in a humid pampean grassland. Proceedings of the $\mathrm{V}^{\text {th }}$ International Rangeland Congress, Utah, USA, 1995: 91-92.

Danais M. 1982. La diversité en Ecologie: analyse bibliographique. Botanica Rhedonica, 17: 77-104.

Djègo JG. 2006. Phytosociologie de la végétation de sous-bois et impact écologique des plantations forestières sur la diversité floristique au sud et au centre Bénin. Thèse de Doctorat en Gestion de l'Environnement, Ecole Doctorale Pluridisciplinaire "Espaces, Cultures et Développement", FLASH, Université du Bénin, 359 p.

Djègo J, Oumorou M. 2009. Phytosociologie de sous-bois et impact des plantations forestières sur la diversité floristique dans la forêt classée de la Lama. Annales des Sciences Agronomiques du Bénin, 12(1): $35-54$.

FOA. 2011. Situation des forêts du monde. ISBN, 978-92-5-206750-4 ; Rome, Italie, $176 \mathrm{p}$.

Lacoste, Roux. 1972. L'analyse multidimensionnelle en phytosociologie et en écologie. Application à des données de l'étage subalpin des Alpes maritimes.
II. L'analyse des données écologiques et l'analyse globale. Oecol. Plant, 5(2): 125146.

Mariot A, Mantovani A, dos Reis MS. 2007. Developing a basis for management of natural populations of Drimys brasiliensis in Brazil, used for its bark. Multiple Use Management of Natural Forests and Woodlands: Policy Refinement and Scientific Progress, Bester JJ, Seydack AHW, Vorster T, Van der Merwe IJ, Dzivhani S (eds). Department of Water Affairs and Forestry: Pretoria, South Africa; 76-81.

ORSTOM. 1968. Carte des sols de la station de Niaouli au 1/5000. Notice explicative. Rapport annuel 3ème partie. ORSTOM. Paris, France, 14p.

Paradis G, Houngnon P. 1977. La végétation de l'aire classée de la Lama dans la mosaïque forêt-savane du Sud-Bénin (ex Sud-Dahomey). Bull. Mus. Nat. Hist. Nat. Botanique, 34: 169-196.

Sinsin B, Hessou C, Houessou L, Lougbegnon T, Niyonkuru C, Mama A. 2004. Etude de la biodiversité dans l'aire d'intervention du projet de gestion des forêts et terroirs riverains au Bénin. Rapport synthèse, DFRN/MAEP, Cotonou, Bénin, 47 pages + annexes.

Sinsin B, Djègo J, Houngbo E, Houéhounha $\mathrm{R}$, Lougbégnon T, Orékan V, Tchtintchin Q. 2010. Etude de la diversité biologique dans les périmètres de reboisement de Kilir et d'Abomey appartenant à l'aire d'intervention du PGFTR au Bénin. Rapport d'étude LEA-FSA/PGFTR, Bénin, 110p. + Annexes.

Sokpon N. 1995. Recherches écologiques sur la forêt dense semi-décidue de Pobè au Sud-Est du Bénin: groupements végétaux, structure, régénération naturelle et chute de litière. Thèse de doctorat, Université Libre de Bruxelles, 365p. 